\title{
Internet of Vehicle Layered Architecture and Network Model
}

\author{
Sheemaa Ali Mohamed Hashim', Rudzidatul Akmam Dziyauddin², Mohd Azri Bin Mohd Izhar ${ }^{3}$, \\ Suriani Mohd $\mathrm{Sam}^{4}$, Norliza Mohamed ${ }^{5}$ \\ ${ }^{1}$ Razak Faculty of Technology and Informatics, UniversitiTeknologi Malaysia (UTM), Malaysia- \\ Computer Science Department, College of Arts and Science in KhameesMushait, King Khalid University, Saudi Arabia, \\ eng_sheemaali@hotmail.com \\ ${ }^{2,3,5}$ Razak Faculty of Technology and Informatics, UniversitiTeknologi Malaysia (UTM), Malaysia \\ ${ }^{2}$ rudzidatul.kl@utm.my, ${ }^{3}$ mohdazri.kl@utm.my, ${ }^{5}$ norlizam.k1@utm.my \\ ${ }^{4}$ Advanced Informatics School, UniversitiTeknologi Malaysia (UTM), Malaysia, suriani.k1@utm.my
}

\begin{abstract}
Nowadays most people and manufacturers willing to facilitate their life by applying the new technology, which is the Internet of Things (IoT). IoT is a multidisciplinary model that allows and grants everything with connectivity property. Internet of Vehicles (IoV), a modern IoT, is a communication system between vehicles and networks, which boosts the vehicles to be intelligent by connecting to the internet and providing different mobile application services. This paper reviews the prevalent and emerging IoV paradigms and network layered architectures, with an emphasis on their functions, and main elements in the network model. Then how the IoV ecosystem works as a network model from sensing, gathering, storing and processing the collected information, and finally accessing the service is proposed in a new Contemporary architecture of the IoV model (CIoV). CIoV consists of four layers namely client layer, communication layer, a cloud layer, and cater a service layer. $\mathrm{CIoV}$ is approached in light of the main functions of the IoV ecosystem, which can be implemented for different and heterogeneous communication models. Finally, based on a case study the CIoV protocol stack is discussed for each layer in the architecture by considering operational and security planes.
\end{abstract}

Key words: InternetofThings(IoT), InternetofVehicle (IoV), Network layered architecturesContemporary IoV (CIoV).

\section{INTRODUCTION}

In recent years and with the rapid progress in technologies, the Internet of Things (IoT) is important for a wide range of scientific and industrial processesand research. Aspect to the future of the Internet, IoT will comprisemillionsof intelligent communicating 'things' through using the Internet, and the connectivity will be in anything, anyone, anywhere and anytime[1]. With increased sophistication in theIoT, it is expected that about 50 billion devices will be interconnected by2020andtrafficdataflowwillgrow 1000 times than before[2].
IoT comprises of sensors, network communicating devices, web storage and data processing, and the applications. Sensors sense and collect the required data. Network components transfer and store the information on cloud servers. Web storage toolsapply data mining methods and techniques on voluminous data to derive valuable information. Then by using an application on a physical device, the processed information will utilize [3]. Devices availability in the IoT which will be related to the connectivity of those devices must be fulfilled in the hardware andsoftware planes to provide anytime and anyplace services for customers. The ability of IoT applications indicates as software availability will provide the services for every person at different places and at the same time. Meanwhile, hardware availability indicates the presence of the devices all the time[4].

The future of the Internet will consist of heterogeneously connected devices (stationary and mobile devices) moreover, this will sustain the borders of the world with physical entities and virtual components. Recently, a major paradigm shift has been on the way toward using IoT in the vehicular domain, e.g., vehicle-to-person (V2P), vehicle-tovehicle (V2V), vehicle-to-sensors (V2S), vehicle-toroadside (V2R) and vehicle-to-transportation infrastructure (V2I), also referred to Vehicular-toEverything (V2X)[5].

To work on the IoV ecosystem and provide applications or services to vehicles/users, the procedure of the system starting from the vehicle upto getting the services should be understood. IoV moves the control of connecting, managing and processing from humans to autonomous vehicles[6].With the high ability of these vehicles, the disposal will be much better and faster than the human and decision will be best in many cases, such as:if earthquake happens, the vehicle will be able to check the affected area and which roads are empty and consequently also alert other vehicles or calling emergency ambulance[7]. 
Thus far, this paper proposes an exhaustive and full IoV network paradigm, with the assertion on a layered architecture. The proposed framework has considerable capability to supply dependable vehicular communications. The main contributions of thispaper are asfollows.

- A network model is proposed by determining four network elements of IoV including user, connected vehicle, network connection, and cloud computing.

- AContemporaryIoV(CIoV)fromfour-layered architectures is proposed focusing on functionalities, operationa land representations of the layers. A protocol stack for the layered architecture is constructed taking into account, operational and securityplanes.

- Visualization of IoV benefits andchallenges

The overall structure of the paper takes the form of eightsections, sort out as follows. Section 2 presents the network environment almodel of IoV. Different proposed layered architectures are derived in Section 3. In Section 4, new Contemporary IoV architecture introduced. The protocol stack structure presented in Section 5. Section 6 set out a case study about the proposed CIoV. And the challenges of the networklayeredmodelinIoVareinscribedinsection 7 . Finally, the most obvious finding to emerge from this study is concludedin Section 8 .

\section{ENVIRONMENTOFIOVNETWORKMODEL}

The IoV network model is a full descriptive model of the communication system in the IoV environment. The network environment model as shown in Figure (1), illustrates the interactions and communications between different elements and networks in the system such as V2V, V2R, V2S and V2I [8].

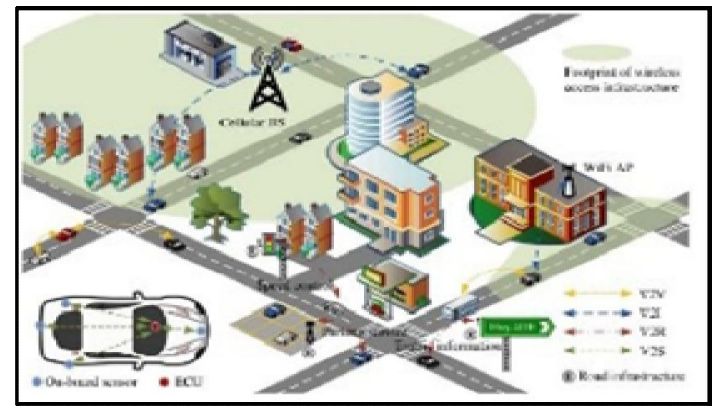

Figure 1: IoV network environment model [9].

IoV network model has four major elements: user, connected-vehicles, network connection, and cloud computing, illustrated in Figure 2.

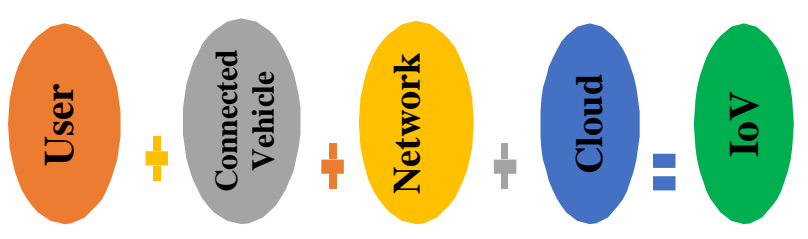

Figure 2: IoV elements.

The user is the beneficiary of the IoV services maybe a driver or any person in the road connected to the vehicle or another vehicle. IoV can provide many services to the users such as aiding the driver in route information including public purposes likecongestion informationandreducingairpollution, andemergency purposes like calling emergency vehicles in accident cases $[10,11]$.

Connected vehicles refer to the epochal vehicles contained many sensors and look forward to reaching 200 sensors per vehicle by 2020 [10]. Those sensors offer the data collection function ality such as detecting road conditions, driver habits and autonomous control [12]. With the growth of industrial, nowadays different automobile manufacturers have two options for supporting the internet to the vehicles: brought-in and builtin.Inbrought-inconnectivity, cars can connect to the internet through user smartphone networks via wires (e.g., Universal Serial Bus (USB)) or wirelessly (e.g.,wireless fidelity (Wi-Fi) or Bluetooth) so that the vehicle achieves direct access to the Internet and also extra benefits by using smartphone applications.Built-in connectivity supports the cars with the internet by consolidating the on-board system with cellular service, which lets the cars connect to the Internet through the cellular network such as Fourth Generation-Long Term Evolution (4G-LTE). As a comparison built-in option offers a strong connection to the vehicles and passengers, but the cellular connectivity cannot be changed when established[9, 13, 14].

The third important element of IoV is a network connection, and this explains how other elements in the IoV ecosystem can be connected together by using one of the network technologies.IoV can be connected to the short-range communication networks, such as Bluetooth and ZigBee in the intravehicle communication (i.e. inside the car; e.g. V2S) and for long or medium-range communication network for inter-vehicle communication (i.e. outside the car, e.g. V2V, V2I, V2R, and V2P) such as Wi$\mathrm{Fi}$, Wireless access in vehicular environment (WAVE), Ultra-Wideband (UWB), 3rd-Generation (3G), 4G/LTEand 5G[15], which that $5 \mathrm{G}$ will be the suitable one for IoT services [16].According to the mobilityof the vehicle,themost prevalent technology 
used in intra-vehicle communication is the vehicle ad-hoc network (VANET). The excellent feature of VANET is a self-organized communication network whereby the pre-infrastructure network is not required as the vehicle is considered as a wireless access point (AP). Furthermore, the full infrastructure I supdated periodically depending on the position of the vehicles. The driver/vehicles can get updated information about the new external environment from some hints, such as red lights[10, 17].

The last element is the cloud, which is the brain of the system. The functions of the cloud are numerous including intelligent decision making, computing processing, analysis of the payoff services to modify or update the applications using different tools for critical analysis such as flowchartand graphs, etc.[6, $10,18]$.

\section{IOVNETWORKLAYEREDARCHITEC TURE}

Many studies conducted on "how heterogeneous networks actuate in IoV ecosystem with different functions and jobs grouped and designed in network layered architecture". Turning now to sixth different approaches proposed from different aspects of three, five and seven network layered architectures.

Nanjie et al. approached three-layeredarchitecture (client, connection, cloud) for Intelligent Transport Systems(ITS)asIoVtechnologyinEuropeandJapan. The client layer represents whole sensors and devices inner and exterior the vehicle, responsible for gathering information about the vehicle, thedriver status and events occurred around vehicles, then sending the information to the second layer (connection). The connection layer ensures the communication between all heterogeneous networks and models (i.e. V2V, V2R, V2P, V2I) sending the data to the third layer (cloud). In the cloud layer, all functions are processed and computed to satisfy the applications in the IoV ecosystem [19].

Wan et al. also approached a three-layered architecture (vehicular, location and cloud) the work of each layer similar to, i.e. vehicular layer the same as client layer, location layer represents connection layer and cloud layer like cloud layer. But the bottleneck is that used short-range communication although it can be provided for long-distance communications by connecting far vehicles and infrastructure networks via neighboringvehicles[20].

Also, Gandotra et al. proposed three-layered architectures (area network, network management, and D2D applications) for only Devices to Devices (D2D) communications, without sending any information through the network base station (BS). All devices are represented and communicated together via a direct link in the first layer (area network). In network management the D2D data are accumulated and sent to the core network named (D2 Dapplications layer), to provide the selected application such as public safety and security services,etc. [8].

On the other hand, Kaiwartya et al.proposed fivelayered architecture (perception, coordination, artificial intelligence (AI), application and business). The perception layer collects the information fromthe different actuators in the area like sensors inside the vehicle (e.g. speed, direction and position of the vehicle and the driver attitudes) and outside the vehicles (e.g. traffic environment and weather condition) and sends data to the coordination layer securely. The coordination layer ensures that information gathered from the perception layer is transferred for processing in the artificial intelligence securely and in unified structure, as the latter is collected from different heterogeneous networks such as wireless access in vehicular environment (WAVE), Wireless-Fidelity (Wi-Fi), fourthgeneration/Long term evolution (4G/LTE) and fifthgeneration $(5 \mathrm{G})$. The $(\mathrm{AI})$ layer is the brain of the IoV ecosystem symbolized by the virtual cloud substructure. It is in charge of dealing with the information from the coordination layer and analyzed this aggregating information by using decision-making algorithms. It manages many services in the cloud environment depending on the critical analysis of the information received.The application layer forms the smart applications that finding out from the AI layer to the end-users. The upper layer is a business model that analyzes the usage data of the smart applications by using different strategies such as use case diagram,graphs, differentiationtables, flowchart, etc., to evaluate the budget preparation for managing the applications [10].

However, Juan Contreras-Castillo et al.proposed seven-layered architecture (user interaction, acquisition, pre-processing, communication, management, business, and security). The job of the user interaction layer is to provide asmart interface for the users inside the vehicle, this interface monitors the area outside the vehicle to get information such as traffic, route condition, and car parking. Inside the vehicle, the interface monitors the behavior of the driver to display the best action in the interface based on the current case. The main benefit of the interface is to reduce driver omissions to make drivingsafe. The liability of the acquisition layer is deciding how to collect the different data and information from inside and outside the vehicles and how to send it by using the suitable network 
technology (short-range technology for inside the vehicle e.g. Bluetooth or ZigBee, and for outside transmission long-range technology e.g. Wi-Fi or ultra-wideband). The pre-processing layer analysis the collected information and filters them to avoid the dissemination of unrelated data. The main purpose of Communication layer is to decide how the devices will communicate together to send the gathered information, i.e. how to select the best network from different heterogeneous networks by using intelligent technologies such as fuzzy algorithms, which work on several parameters such as Quality of services (QoS), privacy and security information. The main job of the Management layer is to manage the data transformed among the different elements in the IoV ecosystem. To get better services from the IoV system Business model must be designed in the Business layer depending on processing and analyzing all the coming information from other layers by using differentstatisticaland crucial tools such as flowchart with various types of cloud computing substructure.The last layer (Securitylayer) interacts with all layers to ensure that all functions and information transfer are done in a securemanner [15].

Another work by li-minnn et al. proposed universal IoV (UIoV) architecture also consists of sevenlayered (Identification Layer, Physical Objects Layer, Inter-Intra Devices Layer, Communication Layer, Cloud Services Layer, Multimedia \& Big Data Computation Layer and Application) for smart cities. Identification Layer responsible for uniquely identify each object in the system by giving Naming and Addressing. The main job of Physical Object Layerto pick up all the data from other objects and send them to the Inter-Intra Devices Layer for more processing. The Inter-Intra Devices Layer is a new and unique layerof UIoV that works with the Communication Layer to provide the inter communications between all actors in the system such as V2I, V2P, V2V, V2R, V2S, and V2D. The cloud layer responsible for all computing environments such as software and hardware infrastructure and processing platforms to grantthe scalability of IoV applications and services. The Multimedia and Big Data Layer is a new and exclusive layer in the UIoV, which be composed of three sublayers: Data Pre-processing, Big Data Computation, andIntelligentTransportsub-layers.All the computation for intelligent transportation overall UIoV layers is the main responsibility of the
Multimedia and Big Data Layer. The last layer of the $\mathrm{UIoV}$ is the application layer that performs brilliantservices to end-users based on relevant information from the upper layer, also it defines a group of protocols for transmitting the message[21].

Generally, from the above reviews, it can be concluded that in respect of model layer similar functionalities must be executed, and this is clarified in Table I.

\section{PROPOSED ARCHITECTURE OF IOV}

It has been shown from this review that to enjoy the services or smart application in the IoV ecosystem, the functionalities of the IoV as follows: firstly data is collected by one of the sensors inside or outside the vehicle. Next, the data is disseminated to other elements in the environment by connecting to one of the network technologies according to the type of communication; inside or outside the vehicle. The vehiclei sconnected to cloud computing to process the data. Finally, the best decision by using one of the artificial intelligence algorithms is selected to provide smart service from the vehicle to the user/vehicle. In order to recognize these functionalities, The present study was designed to exemplary them as a group of layers in new proposed universal architecture for the IoV named Contemporary IoV (CIoV), which will be applied as powerful platforms for different and heterogeneous communication models. There are four main layers within this architecture which are: Client layer, Communication layer, Cloud layer, and Cater Serviceslayer.Figure. 3 shows a diagram of the CIoV layers and their main functions.

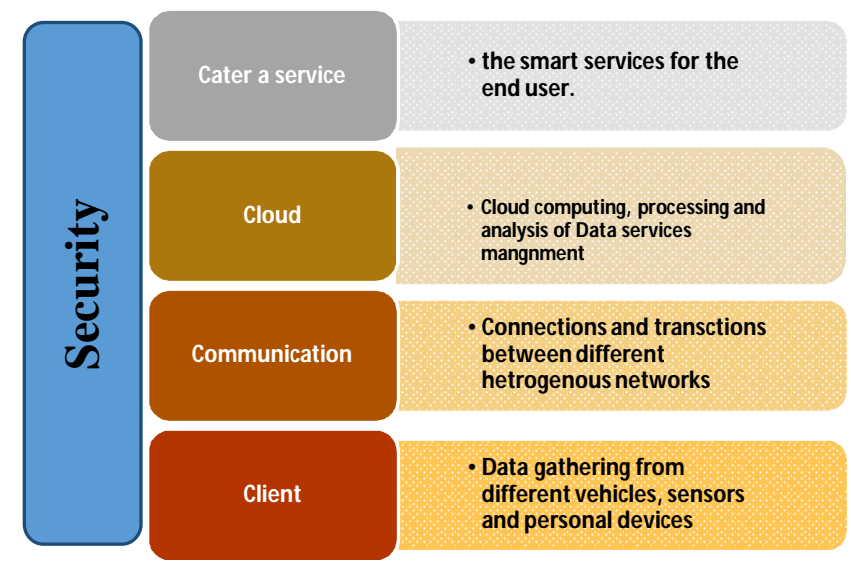

Figure 3:Proposed CIoV 
Table 1: Different Functions of the Network Layered Architecture in IoV

\begin{tabular}{|c|c|c|c|c|c|c|}
\hline \multirow{3}{*}{$\begin{array}{l}\text { IoV } \\
\text { Functions }\end{array}$} & \multicolumn{6}{|c|}{ IoV Network Layer Architecture } \\
\hline & \multicolumn{3}{|c|}{$\begin{array}{l}\text { Three } \\
\text { Layers }\end{array}$} & \multirow{2}{*}{$\begin{array}{l}\text { Five Layers } \\
{[8]}\end{array}$} & \multicolumn{2}{|c|}{ Seven Layers } \\
\hline & {$[16]$} & [17] & [6] & & {$[13]$} & {$[18]$} \\
\hline $\begin{array}{l}\text { Collect } \\
\text { Data from } \\
\text { IOV } \\
\text { actuators }\end{array}$ & \multirow[t]{2}{*}{ Client } & \multirow[t]{2}{*}{ Vehicular } & \multirow{3}{*}{$\begin{array}{l}\text { Area } \\
\text { network }\end{array}$} & \multirow[t]{2}{*}{ Perception } & Acquisition & $\begin{array}{l}\text { Identification } \\
+ \text { object }\end{array}$ \\
\hline $\begin{array}{l}\text { Disseminat } \\
\text { e Data } \\
\text { between } \\
\text { different } \\
\text { actuators } \\
\end{array}$ & & & & & Preprocessing & $\begin{array}{l}\text { Inter-Intra } \\
\text { Devices }\end{array}$ \\
\hline $\begin{array}{l}\text { Connect to } \\
\text { the best } \\
\text { Network } \\
\text { technology }\end{array}$ & $\begin{array}{l}\text { Connectio } \\
\mathrm{n}\end{array}$ & $\begin{array}{l}\text { Locatio } \\
n\end{array}$ & & $\begin{array}{l}\text { Coordinatio } \\
\mathrm{n}\end{array}$ & $\begin{array}{l}\text { Communicatio } \\
\mathrm{n}+ \\
\text { management }\end{array}$ & $\begin{array}{l}\text { Communicatio } \\
\mathrm{n}\end{array}$ \\
\hline $\begin{array}{l}\text { Process the } \\
\text { data in the } \\
\text { Cloud area }\end{array}$ & \multirow[t]{2}{*}{ Cloud } & \multirow[t]{2}{*}{ Cloud } & $\begin{array}{l}\text { Network } \\
\text { managemen } \\
t\end{array}$ & AI & \multirow[t]{3}{*}{ Business } & $\begin{array}{c}\text { Cloud + } \\
\text { Multimedia } \\
\text { and Big Data }\end{array}$ \\
\hline $\begin{array}{l}\text { Provide } \\
\text { smart } \\
\text { service }\end{array}$ & & & $\begin{array}{l}\text { D2D } \\
\text { application }\end{array}$ & Application & & Application \\
\hline $\begin{array}{l}\text { Analysis of } \\
\text { the Usage } \\
\text { of Data }\end{array}$ & $\mathrm{x}$ & $\mathrm{X}$ & $\mathrm{x}$ & Business & & $\begin{array}{l}\text { Multimedia and Big } \\
\text { Data }\end{array}$ \\
\hline Security & $\begin{array}{l}\text { As a } \\
\text { service }\end{array}$ & $\begin{array}{l}\text { Cross } \\
\text { layered }\end{array}$ & $\begin{array}{l}\text { Not } \\
\text { specified }\end{array}$ & $\begin{array}{l}\text { Security } \\
\text { plane }\end{array}$ & As a layer & Cross layered \\
\hline
\end{tabular}

Consistent with the literature, $\mathrm{CIoV}$ supports previous functionalities to enjoy a service in the IoV ecosystem. CIoV will be detailed in the next parts as an operational and security plane. For the operational plane, it is a description of the major functions in each layer in the architecture, and for the security plane will work as across layer means security function will be as a protocol in each layer. Then the protocol stackwill describe in the next section. CIoV will consolidate the different network characteristics regarding the accuracy, interoperability, scalability and has the capability to connect vehicles to heterogeneous networks. The following is a detailed description of each layer.

\subsection{CLENT LAYER}

The top layer of the CIoV architecture is the Client layer, which constituted of diverse types of sensors and actuators inside and outside the vehicles, roadside units (RSUs), and further personal devices. The major liability of this layer is to gather data and information about the vehicle and traffic environment also about other devices in the ecosystem. Most of the informationincludes velocity, position, motor status and journey archive related to the vehicle, on-road vehicle traffic and climate conditions concerning traffic environment, and multimedia and documentary enrollments regarding people. so The considerable responsibility of the layer after collecting this information is to differentiate it in an efficacious and secure manner and send it to the Communication layer.

\subsection{COMMUNICATION LAYER}

The secondary layer of the $\mathrm{CIoV}$ architecture is symbolized by a Communication model fordifferent heterogeneous networks such as Wi-Fi, WAVE, 4G/LTE, 5G, mmWave and satellite networks to provide fast and reliable connections between different actuators in the system, and over those networks, the collected information from the first layer will transfer to the cloud layer forprocessing. 
First of all, the selected network will depend on the connection types is it inter-vehicular or intra-vehicular. For inter-vehicular will be long-range communication because it will be outside the vehicle, and the best network technology will be mmWave technology because it will be suitable for high mobility of the vehicle and will satisfy the expected high data rate services[22]. Intra-vehicular will be one of short-range communications such as Bluetooth or Zigbee and this no need high-frequency range because it will not affect by the mobility of the vehicle. Over the past decade, the selection of a suitable network depends on the assessment of single property such as Received Signal Strength or Signal to Interference Noise [15, 23, 24]. According to [25]the selection depends on the requirements of the service and the network availability. The most challenge was to design asmart technique that selects the most convenientnetwork depending on some related information such as vehicle velocity and beamforming techniques.

\subsection{CLOUD LAYER}

The third layer ofthe CIoV architecture is symbolized by intelligent cloud infrastructure. The main responsibility of this layer is dealing with the informationgatheredfromthelower layer; saving and analyzing them and make a decision based on the critical analysis. According to [10], the primary componentsof this layer are computing and analysis techniques such as ExpertSystem, Vehicular Cloud Computing(VCC), and Big Data Analysis (BDA).

\subsection{CATER A SERVICE LAYER}

The last layer of the CIoV architecture is a Cater a Services layer, which appears as a smart application to provide safety and non-safety services to the vehicles and end-users in an efficient manner. This layer is based on a rational and accurate analysis of the processed information by the Cloud layer.

\section{CIOV PROTOCOLSTACK}

The Protocol stack of the $\mathrm{CIoV}$ architecture is discussed as two-planes (operational and security) to aid the current protocols in the various layers. The protocol stack is based on several projects for vehicular networks which include WAVE [26], Car-2-Car (C2C)[27], and Communication Architecture for Land Mobile (CALM) [28, 29],and projects for IoT including HyDRA [30], IoT6 and IoT-A[31].

The operational plane explained the protocols used in the four layers of the CIoV. The client layer implicates different ways to be in contact with the driver and trippers, such as attributes, voice and visual which covers physical layer connections and example of suitable protocols Worldwide Interoperability for
Microwave (WiMax) [32], Ethernet, 4G/Long Term Evolution (LTE) [33],satellite communications and Global System for Mobile communication (GSM)[34]. In the communication layer, active and outlet protocols are categorized into two transmission classification:Intra- vehicular and inter-vehicular. Intravehicular means the communication inside the vehicle such as NearField Communication, Bluetooth,IEEE 1609, Radio Frequency Identification, WAVE, ultra-wideband, and WiFi. Inter-vehicular includes all protocols to communicate outside of the vehicle such as2G/3G/4G/LTE/5G, IEEE802.15.4,802.11p,WiMAX, Low-Power Wide Area Network and mmWave. The protocols in the Cloud layer, are new research issues and challenges in IoV due to the inaccessibility of appropriate protocols for Vehicular Cloud Computing (VCC) and Big Data Analysis (BDA), some related protocols in this layer such as CALM Service Layer (CALM-SL) and WAVE-1609.6 service-related protocols[15].In the Cater Services layer, an example of one asset managerprotocol 1609.1 defined in WAVE used for controlling the resources by using smart and brilliantinterfaces and applications[10].

The main goal of the security plane is to hold up all securityfunctions in the $\mathrm{CioV}$ as a cross-laye rover the four layers. Some examples of the works security protocols in the IoV ecosystem are: The security information connector designed in the WAVE project, Hardware Security Module proposed in the CALM projectand the Security Management Information Base sophisticated based on the C2C project [10].

\section{CIOV CASE STUDY}

This section will explain CIoV as a case study, assume that the Ambulance vehicle wants fast connections in the IoV ecosystem for an emergency case. The steps will be as follows, and Figure 4 illustrates it:

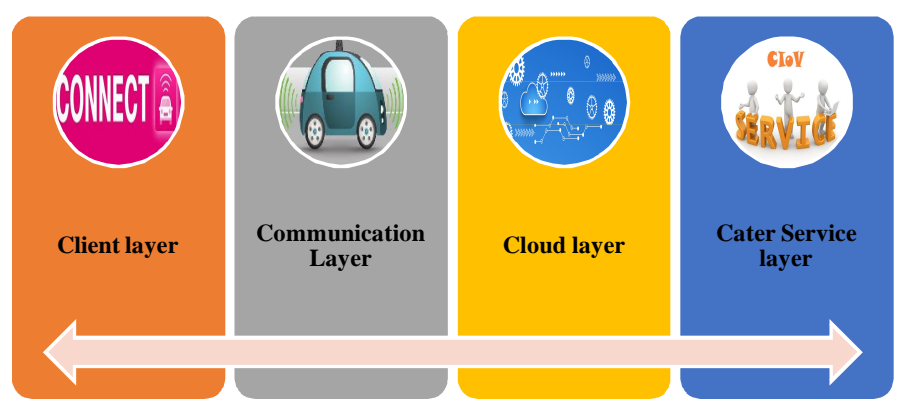

Figure 4: CIoV case study

- The vehicle sensor forms the client layer will collect information about the need of the Ambulance vehicle to communicate using a 
standard protocol such as Ethernet and start to alert the signal to disseminate the data such as vehicle velocity to a nearby base station (BS).

- As a part of the communication layer, the client will select the suitable access technology for data dissemination and begin the broadcasting process to the BS using a standard protocol such as mmWave technology. The received BS notifies the vehicle to start the connection.

- All the data and knowledge that disseminated in the system stored and analyzed in the cloud layer using a standard protocol such as BDA or VCC to process reports about the case which will be useful in other similar cases and overcome future connection and service failures.

- Finally, the vehicle gets the service which is a connection to the hospital unit through any user interface inside the vehicle, and all the data will be transmitted in a secure manner (security layer).

\section{IOV CHALLENGES}

A key issue of the $\mathrm{IoV}$ is to provide the best and reasonable communications between different users, vehicles and heterogeneous networks. Obviously,IoV has several and special issues, tasks and challenges must be addressed, most of them questioned as which network model should be selected, which network technology will be suitable for communications with moving vehicles, how different actuators in the same network environment will participate and how smart serviceswillbeofferedtovehiclesandusers. Onthe face of these questions, we put forward the most challenges as follow:

Technological challenges:Most studies in the field of IoV have only focused on its applications, not the deployment and how to implement the whole system.However, there are technological challenges for deployment in smart cities, such as the combinations of all components and communications items[21].

Big data challenges: Another challenge isthe fast development in the vehicle and the different objects connected to the vehicles. Also, the huge data collections between different actuators in the ecosystem resulting in the challenge and research gaps in the analysis and processing of this Big data. There is less research considering the Big data in the IoV system, but the recent work[21], introduces a unique layerin their architectures named Multimedia and Big Data, focused on Big data aggregations, processing, and analytics.

Connectivity challenges: The mostimportant challenge is how to select the best network for connections from heterogeneous networks based on the vehicle and environmentstatustoimprovetheconnection ability inreal-time[35].

Security challenges: the dynamic environment of the IoV ecosystem with different heterogeneous networks and the increasing number of vehicles requires the vehicle to validate the connected networks and receiver inreal-time[10].

\section{CONCLUSION}

Internet of Vehicles (IoV) is formulating from heterogeneous vehicular networks to achieve the concept of smart transport by connected vehicles. Overall,this paper contributes to anexhaustive structure of IoV. Successively, it will be a basis to acquire the overall understanding of the layered architecture, network model and challenges of IoV. Followed by proposed Contemporary IoV (CIoV) architecture, consisting of four layers, client layer, communication layer, cloud layer and cater services layer. CIoV covers the four main functions of the IoV ecosystem, to allow combination and transaction between different heterogeneous objects and networks. Then a brief explanation of the protocols tack for each layer is described, and a case study was added for more explanation.Finallydetailed about IoV challenges have been discussed.

\section{ACKNOWLEDGMENTS}

This work is supported by the Ministry of higher education under the grant R.K130000.7856.5F277for the publication. We would like to extend our gratitude to U-BAN members for their comments on the work.

\section{REFERENCES}

[1] D. R. L. S. Mahalakshmi1, "Artificial Intelligence with the Internet of Things on Healthcare systems: A Survey," International Journal of Advanced Trends in Computer Science and Engineering, vol. Volume 8, no. No.6, pp. 2847 - 2854, November - December 2019.

[2] O. Bello and S. Zeadally, "Intelligent device-to-device communication in the internet of things," IEEE Systems Journal, vol. 10, no. 3, pp. 1172-1182, 2014.

[3] R. Gupta and R. Gupta, "ABC of Internet of Things: Advancements, benefits, challenges, enablers and facilities of IoT," in Colossal Data Analysis and Networking (CDAN), Symposium on, 2016, pp. 1-5: IEEE.

[4] A. Al-Fuqaha, M. Guizani, M. Mohammadi, M. Aledhari, and M. Ayyash, "Internet of things: A survey on enabling technologies, protocols, and applications," IEEE Communications Surveys \& Tutorials, vol. 17, no. 4, pp. 2347-2376, 2015.

[5] T. I. B. Yung-Fa Huang1, Hui-Yu Huang2, Wen Huang1, "V2V Routing with Fuzzy Inference Mechanism in 
Vehicular Networks," International Journal of Advanced Trends in Computer Science and Engineering, vol. Volume 9, no. No.1, pp. 74 - 79, January - February 2020.

[6] M. Gerla, E.-K. Lee, G. Pau, and U. Lee, "Internet of vehicles: From intelligent grid to autonomous cars and vehicular clouds," in 2014 IEEE world forum on internet of things (WF-IoT), 2014, pp. 241-246: IEEE.

[7] E. Sdongos, A. Bolovinou, M. Tsogas, A. Amditis, B. Guerra, and M. Manso, "Next generation automated emergency calls," IEEE CCNC (Globe-IoT, 2017). Las Vegas, Nevada, USA, 2017.

[8] P. Gandotra, R. K. Jha, and S. Jain, "A survey on device-todevice (D2D) communication: Architecture and security issues," Journal of Network and Computer Applications, vol. 78, pp. 9-29, 2017.

[9] N. Lu, N. Cheng, N. Zhang, X. Shen, and J. W. Mark, "Connected vehicles: Solutions and challenges," IEEE internet of things journal, vol. 1, no. 4, pp. 289-299, 2014.

[10] O. Kaiwartya et al., "Internet of vehicles: Motivation, layered architecture, network model, challenges, and future aspects," IEEE Access, vol. 4, pp. 5356-5373, 2016.

[11] K. Zheng, Q. Zheng, P. Chatzimisios, W. Xiang, and Y. Zhou, "Heterogeneous vehicular networking: A survey on architecture, challenges, and solutions," IEEE communications surveys \& tutorials, vol. 17, no. 4, pp. 2377-2396, 2015.

[12] M. Pinelis, "Automotive Sensors and Electronics: Trends and developments in 2013 in Automot," Sensors Electron. Expo, Detroit, MI, USA, 2013.

[13] R. Hussain and S. Zeadally, "Autonomous cars: Research results, issues, and future challenges," IEEE Communications Surveys \& Tutorials, vol. 21, no. 2, pp. 1275-1313, 2018.

[14] R. Copeland, "Automotive context-aware policy system for car connectivity requests," in 2015 18th International Conference on Intelligence in Next Generation Networks, 2015, pp. 128-135: IEEE.

[15] J. Contreras-Castillo, S. Zeadally, and J. A. Guerrero Ibáñez, "A seven-layered model architecture for Internet of Vehicles," Journal of Information and Telecommunication, vol. 1, no. 1, pp. 4-22, 2017.

[16] A. B. 1Radhia Khdhir "5G LTE-A Cognitive Multiclass Scheduling Scheme for Internet of Things," International Journal of Advanced Trends in Computer Science and Engineering, vol. Volume 8, no. No.5, pp. 2485 - 2491, September - October 2019.

[17] V. Bychkovsky, B. Hull, A. Miu, H. Balakrishnan, and S. Madden, "A measurement study of vehicular internet access using in situ Wi-Fi networks," in Proceedings of the 12 th annual international conference on Mobile computing and networking, 2006, pp. 50-61: ACM.

[18] T. Mekki, I. Jabri, A. Rachedi, and M. ben Jemaa, "Vehicular cloud networks: Challenges, architectures, and future directions," Vehicular Communications, vol. 9, pp. 268-280, 2017.

[19] N. Liu, "Internet of Vehicles: Your next connection," Huawei WinWin, vol. 11, pp. 23-28, 2011.
[20] J. Wan, D. Zhang, S. Zhao, L. T. Yang, and J. Lloret, "Context-aware vehicular cyber-physical systems with cloud support: architecture, challenges, and solutions," IEEE Communications Magazine, vol. 52, no. 8, pp. 106-113, 2014.

[21] L.-M. Ang, K. P. Seng, G. K. Ijemaru, and A. M. Zungeru, "Deployment of IoV for Smart Cities: Applications, Architecture, and Challenges," IEEE Access, vol. 7, pp. 6473-6492, 2018.

[22] B. Antonescu, M. T. Moayyed, and S. Basagni, "mmWave channel propagation modeling for V2X communication systems," in Personal, Indoor, and Mobile Radio Communications (PIMRC), 2017 IEEE 28th Annual International Symposium on, 2017, pp. 1-6: IEEE.

[23] S. Kunarak and R. Suleesathira, "Predictive RSS with fuzzy logic based vertical handoff algorithm in heterogeneous wireless networks," in The 2010 International Conference on Advanced Technologies for Communications, 2010, pp. 189-194: IEEE.

[24] B.-J. Chang, J.-F. Chen, C.-H. Hsieh, and Y.-H. Liang, "Markov decision process-based adaptive vertical handoff with RSS prediction in heterogeneous wireless networks," in 2009 IEEE Wireless Communications and Networking Conference, 2009, pp. 1-6: IEEE.

[25] A. Guerrero-Ibáñez, J. Contreras-Castillo, A. Barba, and A. Reyes, "A QoS-based dynamic pricing approach for services provisioning in heterogeneous wireless access networks," Pervasive and Mobile Computing, vol. 7, no. 5, pp. 569-583, 2011.

[26] I. S. Program, ITS Standards Program, 2016.

[27] C. J. Hill and J. K. Garrett, "AASHTO connected vehicle infrastructure deployment analysis," United States. Joint Program Office for Intelligent Transportation Systems2011.

[28] M. Staubach, N. Schebitz, F. Köster, and D. Kuck, "Evaluation of an eco-driving support system," Transportation research part F: traffic psychology and behaviour, vol. 27, pp. 11-21, 2014.

[29] R. Bossom et al., "D31 european its communication architecture," tech. rep, 2009.

[30] Hydra, Hydra project, 2011.

[31] IoT6.eu., "IoT6.eu Researching IPv6 potential for the Internet of Things," IoT6.eu Researching IPv6 potential for the Internet of Things, 2016.

[32] I. 802.16, "IEEE 802.16 Based Standards. WiMax Forum-Technology,," WiMax Forum-Technology, 2016.

[33] C. Cox, An introduction to LTE: LTE, LTE-advanced, SAE and $4 G$ mobile communications. John Wiley \& Sons, 2012.

[34] G. Maral and M. Bousquet, Satellite communications systems: systems, techniques and technology. John Wiley \& Sons, 2011.

[35] S. Huang, Y. Gao, W. Xu, Y. Gao, and Z. Feng, "Energy-Angle Domain Initial Access and Beam Tracking in Millimeter Wave V2X Communications," IEEE Access, vol. 7, pp. 9340-9350, 2019. 